\title{
Crecimiento de las plantaciones de Eucalyptus globulus sobre suelos rojo arcillosos de la provincia de Osorno, Décima Región
}

\author{
Growth of Eucalyptus globulus plantations on red clay soils in the Province of Osorno, \\ $10^{\text {th }}$ Region, Chile \\ EDITH GELDRES ${ }^{1}$, JUAN E. SCHLATTER ${ }^{2}$ \\ ${ }^{1}$ Departamento de Ciencia y Tecnología Forestal, Universidad de Los Lagos, Casilla 933, Osorno, Chile. \\ E-mail: edgeldres@surnet.cl \\ ${ }^{2}$ Instituto de Silvicultura, Facultad de Ciencias Forestales, Universidad Austral de Chile, Casilla 567, \\ Valdivia, Chile. E-mail: jschlatt@uach.cl
}

\begin{abstract}
SUMMARY
Eucalyptus sp. plantations began to be commercially established in 1990 to 2000 in the $10^{\text {th }}$ Region, or "Región de Los Lagos", Chile, as a result of both a good market and high growth expectations. Further estimations indicated that the growth rates in the Province of Osorno were actually less than those planned, but no measurements were available to back them up. The objective of this work was to measure the volume growth of Eucalyptus globulus plantations established in the western area of the Province of Osorno. The study area was located between $40^{\circ} \mathrm{S}$ to $41^{\circ} \mathrm{S}$ and comprised red clay soils that belonged to the Fresia family, specifically the Cudico and Crucero series. Different plantations, eight years old on average, were measured in areas of at least 5 ha each one, with a total area of 300 ha. These plantations were managed with minimum planting techniques, which meant that they all had to be partially replanted, mainly due to damage caused by frosts during the first two years. A simple random sampling was performed in each plantation with a maximum error of $15 \%$ of the basal area. The volume was estimated with a volume function for the species. The annual average increase of E. globulus in the area reached 10 to $12 \mathrm{~m}^{3} / \mathrm{ha} /$ year, which was the lowest growth level expected, as a result of the lack of adequate establishment techniques and low temperatures during the winter. However, one particular plantation of the same species established in a propitious place of the same area and with better establishment techniques reached a substantial higher growth of $32 \mathrm{~m}^{3} / \mathrm{ha} /$ year. The results obtained indicate that in this area, with marginal climatic conditions for the species, very careful site selection is required and that warmer aspects might be preferred. Intensive establishment techniques must also be practiced such as soil cultivation, weed control and fertilization.
\end{abstract}

Key words: volume growth, red clay soils, Eucalyptus globulus.

\section{RESUMEN}

Las plantaciones de Eucalyptus sp. se comenzaron a establecer en forma comercial en la Región de Los Lagos durante la década 1990-2000, basadas en las buenas expectativas de mercado y de crecimiento. Estimaciones posteriores indicaron que los rangos de crecimiento en la provincia de Osorno fueron en realidad menores a los esperados, pero no se contó con mediciones para respaldarlas. El objetivo de este trabajo fue medir el crecimiento en volumen de las plantaciones de Eucalyptus globulus, establecidas en el sector occidental de la provincia de Osorno. El área de estudio se sitúa entre $\operatorname{los} 40^{\circ}-41^{\circ} \mathrm{S}$, con suelos rojo arcillosos pertenecientes a la familia Fresia, específicamente de las series Cudico y Crucero. Se midieron diferentes plantaciones, en superficies de al menos 5 ha, sumando la población total 300 ha, con un promedio de 8 años de edad. Estas plantaciones fueron efectuadas con las mínimas técnicas de plantación, debiéndose replantar parcialmente en todas, principalmente debido a daños 
BOSQUE 25(1): 95-101, 2004

Crecimiento de las plantaciones de Eucalyptus globulus sobre suelos rojo arcillosos...

por heladas durante los dos primeros años. En cada plantación se realizó un muestreo aleatorio simple con un error máximo del 15\% respecto al área basal. La estimación de volumen se efectuó aplicando una función de volumen para la especie. El incremento medio anual de Eucalyptus globulus en el área de estudio alcanzó con 10-12 m³/haaño, el nivel más bajo del crecimiento proyectado para la Región de Los Lagos, como resultado de una falta de técnicas adecuadas al establecimiento y por las bajas temperaturas del período invernal. Sin embargo, en plantaciones de la misma especie, establecidas en un sitio favorable de la misma zona y con técnicas de establecimiento más exigentes, se logró un crecimiento muy superior: 32 m³/ha-año. Los resultados obtenidos indican que en esta zona, con condiciones climáticas marginales para la especie, se requiere en primer lugar una cuidadosa selección del sitio, considerando exposiciones más cálidas. Además, deberán aplicarse técnicas de establecimiento con cultivo del suelo, control adecuado de malezas y fertilización.

Palabras claves: crecimiento volumétrico, suelos rojo arcillosos, Eucalyptus globulus.

\section{INTRODUCCION}

Plantaciones forestales con eucaliptos se establecieron en las provincias de Osorno y Llanquihue, Región de Los Lagos, durante los años posteriores a 1990, si bien ya existían árboles aislados, en cortavientos o en parcelas experimentales. Entre las especies introducidas destaca Eucalyptus globulus Labill. como también Eucalyptus nitens Maiden, Eucalyptus delegatensis R. T. Baker, Eucalyptus regnans $\mathrm{F}$. Muell. y Eucalyptus viminalis Labill. entre otras. Según Prado y Barros (1), para la Región Oceánica de Los Lagos, delimitada entre los paralelos $39^{\circ}$ y $42^{\circ} \mathrm{S}$, las proyecciones de crecimiento para Eucalyptus globulus spp globulus sería de 10 a $29 \mathrm{~m}^{3} / \mathrm{ha}$-año. Estos datos y las buenas perspectivas de mercado motivaron el establecimiento de plantaciones de Eucalyptus globulus por propietarios privados medianos y empresas forestales que se establecieron en la región. Los primeros resultados de crecimiento obtenidos, sin embargo, indicaron que el rango logrado en las plantaciones de la región, unidad Valdivia (2), fue menor a la proyección señalada por los autores citados. Pero existía una falta notoria de mediciones que respaldara esos resultados. El presente trabajo tiene como objetivo llenar ese vacío y evaluar las plantaciones de Eucalyptus globulus establecidas en la parte occidental de la provincia de Osorno, en terrenos con suelos rojo arcillosos, y así determinar si las proyecciones de crecimiento pueden ser alcanzadas.

\section{MATERIAL Y METODOS}

El área de estudio se sitúa entre los $40^{\circ}-41^{\circ} \mathrm{S}$, equivalente al rango de coordenadas de proyec- ción cartográfica UTM, Este 632 a 644 km y Norte 5.462 a 5.508 km. En esta área se desarrollan plantaciones de Eucalyptus globulus, actualmente con una edad entre 8-9 años, distribuidas entre las comunas de Osorno, Río Negro y Purranque, provincia de Osorno, Décima Región.

También se incorporaron en el análisis algunas plantaciones que escapan a la población principal muestreada, siendo estas tratadas como casos especiales: una plantación de Eucalyptus globulus cultivada con técnicas de establecimiento más exigentes y otras dos, una de Eucalyptus delegatensis y otra de Eucalyptus viminalis, todas de 8 años de edad.

Para toda el área de estudio las características climáticas corresponden al distrito de crecimiento 1-2 (3), el cual se caracteriza por presentar precipitaciones anuales entre $1.400-1.500 \mathrm{~mm}$. El período seco, en que la precipitación no alcanza a cubrir el $50 \%$ de la evapotranspiración potencial, puede ser de un mes al año, con un valor máximo de dos meses al año. El índice de humedad anual varía entre un valor de 2,0 y 2,5 , con un índice de humedad estival variable entre 0,4 y 0,7 , y con una humedad relativa entre un $70-80 \%$. El período libre de heladas fluctúa entre 150-200 días y el número total de heladas oscila entre 20-30 al año. La temperatura media anual es de $11,2^{\circ} \mathrm{C}$, la media del mes más cálido (enero) de $16-17^{\circ} \mathrm{C}$, la media del mes más frío (julio) de $6,5-7,0^{\circ} \mathrm{C}$ y la mínima absoluta para este distrito climático es de $-6^{\circ} \mathrm{C}$ con una frecuencia de un mes al año.

Los suelos corresponden a rojos arcillosos pertenecientes a la familia Fresia, específicamente las series Cudico y Crucero. Estos suelos rojo arcillosos se presentan en una geomorfología de cerros y lomajes, inmediatamente al oriente de la Cordille- 
ra de la Costa, extendiéndose hasta la Depresión Intermedia.

Según el Instituto de Investigaciones Agrarias (4), los suelos fueron formados a partir de cenizas volcánicas pleistocénicas puras o mezcladas con otros materiales de desgaste de la Cordillera de la Costa, sedimentados sobre antiguas morrenas glaciales y/o sobre rocas metamórficas del basamento de la Cordillera de la Costa. Son moderadamente profundos a profundos, de textura franco arcillosa a arcillosa y bien estructurados. La densidad aparente es media, y frecuentemente presentan drenaje restringido en el subsuelo, causado por el cambio textural y la discontinuidad de la densidad entre el suelo y el sustrato morrénico. Los suelos presentan menor profundidad en las cumbres y laderas altas; la profundidad aumenta en las laderas, especialmente hacia su base cuando estas son cóncavas, pero disminuye nuevamente en planos bajos y/o de drenaje natural. Las limitantes que más frecuentemente pueden aparecer son: la profundidad arraigable, de gran variación, y el drenaje interno restringido en el subsuelo; con frecuencia presentan una estructura cerrada y una moderada capacidad de agua aprovechable; acidez y deficiencias de fósforo, potasio y boro; nitrógeno y azufre también son limitantes en terrenos erosionados. Existe una alta susceptibilidad a la compactación (3).

En general las plantaciones de Eucalyptus globulus, en el área de estudio, fueron establecidas en el período 1991-1992, sobre suelos ganaderos de capacidad de uso VI, con técnicas simples. Estas se caracterizaron por una preparación puntual del suelo (taza), con un control manual preplantación de las malezas, sin aplicación de fertilizantes. Además, se efectuaron replantes al segundo y tercer años, principalmente por pérdidas causadas por las heladas y en menor proporción por la competencia de las malezas.

El muestreo se realizó en plantaciones de eucaliptos establecidas en las comunas de Osorno, Río Negro y Purranque, provincia de Osorno, $\mathrm{X}$ Región. De un total de nueve plantaciones se eligió al azar una muestra de cinco plantaciones en los suelos serie Cudico, representando una superficie de 135 ha. De igual modo, de un total de diez plantaciones se eligieron al azar otras cinco en los suelos serie Crucero, representando una superficie de 165 ha. Las repeticiones en cada serie se reali- zaron sobre distritos climáticos iguales; por lo tanto, representan una misma población climática.

El muestreo incorporó como casos especiales para la serie Cudico: un rodal con Eucalyptus delegatensis, un rodal con Eucalyptus viminalis y finalmente un rodal de Eucalyptus globulus; este último, de 5,2 ha, implantado con óptimas condiciones de establecimiento, es decir, con un cultivo del suelo que mejoró las características físicas de este, con una fertilización homogénea en toda la plantación y un control de las malezas pre y postplantación. Además, se debe mencionar que en este rodal no se registraron problemas de heladas, dado que era el sector más alto del predio y con una pendiente no mayor al $15 \%$.

En cada rodal se estableció un muestreo aleatorio simple, con parcelas de $200 \mathrm{~m}^{2}$. En cada parcela se midió el diámetro (DAP) de todos los árboles. La intensidad de muestreo dependió de la variabilidad de cada plantación, pero se fijó como máximo error de muestreo un $15 \%$ para el área basal (cuadro 1). La altura promedio se midió en forma aleatoria, considerando cuatro mediciones por clase diamétrica.

Para la estimación del volumen se utilizó una función para eucaliptos, según Bahamondez et al. (5), la cual entrega el volumen en metros cúbicos sólidos sin corteza para un índice de utilización de $5 \mathrm{~cm}$. Esta función fue validada por funciones locales de empresas forestales y por volúmenes reales obtenidos por cosecha en la región.

El análisis de datos entre series de suelo es a través del incremento medio anual ( $\mathrm{m}^{3} / \mathrm{ha}$-año), dado que es el único parámetro que uniforma la comparación. En todas las plantaciones existieron

\section{CUADRO 1}

Número de parcelas utilizadas para las determinaciones dasométricas.

Plot number selected for growth measurements.

\begin{tabular}{|l|c|c|c|c|c|}
\hline Serie & \multicolumn{5}{|c|}{ Rodal/N $\mathrm{N}^{\circ}$ Parcelas } \\
\hline $\begin{array}{l}\text { Serie } \\
\text { Cudico }\end{array}$ & $1 / 8$ & $2 / 15$ & $3 / 7$ & $4 / 4$ & $5 / 5$ \\
\hline $\begin{array}{l}\text { Serie } \\
\text { Crucero }\end{array}$ & $1 / 16$ & $2 / 14$ & $3 / 32$ & $4 / 12$ & $5 / 10$ \\
\hline
\end{tabular}


BOSQUE 25(1): 95-101, 2004

Crecimiento de las plantaciones de Eucalyptus globulus sobre suelos rojo arcillosos...

replantes, actividad que actualmente no se practica, lo que resultó en edades algo heterogéneas. Se consideraron comparables plantaciones con sólo un año de diferencia en la fecha de establecimiento, por el motivo anterior y debido a que los incrementos promedios fueron muy similares para las edades consideradas.

Para el análisis de los datos, entre las dos series de suelo, se aplicó un análisis de varianza de una vía, del incremento medio anual en volumen, considerando estos datos al cuadrado, para así obtener una distribución normal. La probabilidad de error para aceptar diferencias estadísticas significativas fue de 0,05 .

\section{RESULTADOS Y DISCUSION}

Los resultados obtenidos son entregados en los cuadros 2, 3 y 4 y figuras 1 y 2 . El incremento medio anual promedio del Eucalyptus globulus de ocho años para la serie Cudico fue de $10 \mathrm{~m}^{3} / \mathrm{ha}$ año; en cambio, en la serie Crucero, con plantaciones entre ocho y nueve años, se registró un promedio de $11 \mathrm{~m}^{3} /$ ha-año.

Según el análisis estadístico, el incremento medio anual ( $\mathrm{m}^{3} /$ ha-año) registrado en Eucalyptus globulus para las series Cudico y Crucero no presenta, entre estas series, diferencias significativas (probabilidad de error de 0,05). Tal situación refleja el hecho de que las plantaciones se desarrollan dentro de un mismo distrito climático y, además, permite inferir que las diferencias taxonómicas entre las series de suelo no siempre resultan en diferencias de crecimiento.

Los resultados de crecimiento obtenidos en las mediciones efectuadas en Eucalyptus globulus, establecido el año 1991-1992, corresponden a los valores menores del rango de crecimiento citados por Prado y Barros (1) para la especie en la Región de Los Lagos, pero son muy cercanas al rendimiento indicado por estos autores para el sitio

\section{CUADRO 2}

Resultados obtenidos en Eucalyptus globulus para la serie de suelo Cudico, X Región. Results for Eucalyptus globulus established on the Cudico soil series, X Region.

\begin{tabular}{|c|c|c|c|c|c|c|}
\hline Rodal & Especie & $\begin{array}{c}\text { Superficie } \\
\text { (ha) }\end{array}$ & Edad & $\begin{array}{c}\text { Densidad } \\
\text { (arb./ha) }\end{array}$ & $\begin{array}{c}\text { Area Basal } \\
\left(\mathrm{m}^{2} / \mathrm{ha}\right)\end{array}$ & $\begin{array}{c}\text { IMA } \\
\left(\mathrm{m}^{3} / \mathrm{ha}\right)\end{array}$ \\
\hline 1 & E. globulus & 22,2 & 8 & 1.172 & 15,7 & 9,8 \\
2 & E. globulus & 18,1 & 8 & 1.386 & 20,8 & 13,3 \\
3 & E. globulus & 77,0 & 8 & 1.271 & 21,4 & 14,0 \\
4 & E. globulus & 12,6 & 8 & 1.610 & 13,5 & 7,3 \\
5 & E. globulus & 5,1 & 8 & 1.497 & 11,9 & 5,9 \\
\hline
\end{tabular}

\section{CUADRO 3}

Resultados obtenidos en Eucalyptus globulus para la serie de suelo Crucero, X Región. Results for Eucalyptus globulus established on the Crucero soil series, X Region.

\begin{tabular}{|c|c|c|c|c|c|c|}
\hline Rodal & Especie & $\begin{array}{c}\text { Superficie } \\
\text { (ha) }\end{array}$ & Edad & $\begin{array}{c}\text { Densidad } \\
\text { (arb./ha) }\end{array}$ & $\begin{array}{c}\text { Area Basal } \\
\left(\mathrm{m}^{2} / \mathrm{ha}\right)\end{array}$ & $\begin{array}{c}\text { IMA } \\
\left(\mathrm{m}^{3} / \mathrm{ha}\right)\end{array}$ \\
\hline 1 & E. globulus & 30,2 & 8 & 1.620 & 18,2 & 10,8 \\
2 & E. globulus & 26,9 & 8 & 1.270 & 18,4 & 11,2 \\
3 & E. globulus & 58,1 & 9 & 867 & 15,3 & 9,6 \\
4 & E. globulus & 32,0 & 9 & 1.508 & 22,8 & 13,2 \\
5 & E. globulus & 18,0 & 9 & 1.359 & 18,1 & 10,1 \\
\hline
\end{tabular}


Crucero Nuevo, el más próximo desde el punto de vista geográfico y del sitio, a las plantaciones analizadas en el presente trabajo. El incremento medio anual de $10 \mathrm{~m}^{3} /$ ha-año, entregado por los autores para Crucero Nuevo, corresponde a una plantación de 11 años de edad, es decir, el rendimiento en referencia es incluso más limitado, considerando que el crecimiento volumétrico corriente aún está lejos de culminar a esa edad. Aun así las expectativas de rendimiento esperadas para la especie fueron mayores en la zona; las causas que pueden explicar el menor rendimiento de la especie son las siguientes:

El área de estudio representa el límite sur de desarrollo para la especie en Chile, por las temperaturas mínimas de hasta $-6^{\circ} \mathrm{C}$ y en algunos sitios incluso $-8^{\circ} \mathrm{C}$, especialmente en años extraordinarios, que superan lo indicado para su hábitat natural de $-4^{\circ} \mathrm{C}$ (2). Lo anterior puede ocasionar un alto porcentaje de pérdida de la plantación, lo que obliga a continuos replantes.

Sin embargo, en el cuadro 4 se observa que una plantación de Eucalyptus globulus (caso especial), alcanzó un incremento medio anual de 32,1 $\mathrm{m}^{3} /$ ha-año a una edad de 8 años, para un sitio ubicado en la misma área geográfica que las plantaciones anteriores. Pero esta plantación fue establecida en un sitio de temperaturas más benignas, por su posición y exposición, lo que la favoreció.

Además, Eucalyptus globulus es una especie que, inadecuadamente cultivada, está más expuesta a las intensas pero breves heladas, cuando su desarrollo inicial es débil y aún presenta insuficiente lignificación de sus tejidos. En el sitio especial, indicado en el cuadro 4, se aplicaron adecuadas técnicas de establecimiento (cultivo del suelo, control de malezas, fertilización) hasta tal punto, que no fue necesario efectuar replantes en años posteriores por concepto de pérdida de plantas, ya que la plantación se desarrolló mucho más vigorosa desde el inicio. Plantas más desarrolladas y lignificadas son en general más resistentes a las heladas.

La reflexión anterior plantea de alguna manera que el potencial de crecimiento de Eucalyptus globulus en la zona puede ser mejor, cuando la especie es establecida en condiciones óptimas de sitio y al aplicarse técnicas adecuadas de plantación y manejo postplantación.

El rodal con Eucalyptus delegatensis, a una edad de 8 años, presentó un incremento medio

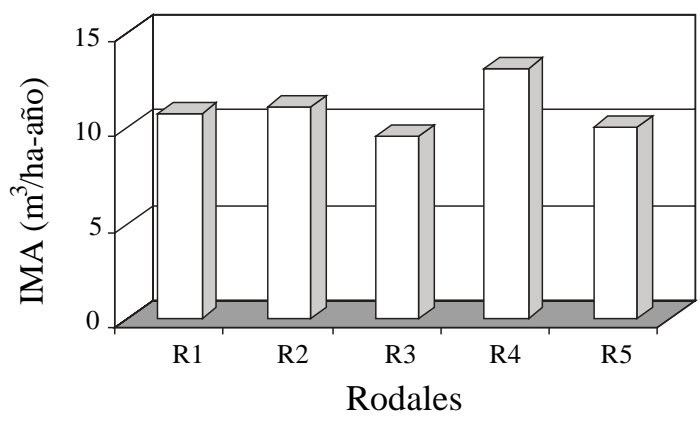

Figura 1. Crecimiento de plantaciones de Eucalyptus globulus en suelos serie Cudico.

Growth of Eucalyptus globulus plantations on Cudico soil series.

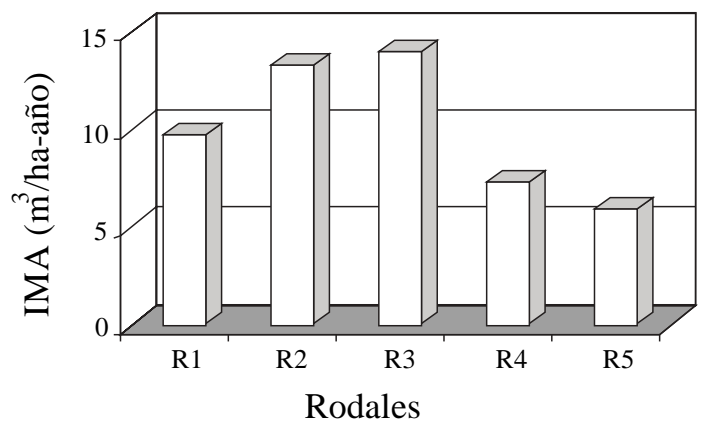

Figura 2. Crecimiento en plantaciones de Eucalyptus globulus en suelos serie Crucero.

Growth of Eucalyptus globulus plantations on Crucero soil series.

anual de $18,1 \mathrm{~m}^{3} / \mathrm{ha}$-año, pero con técnicas de establecimiento simples. No requirió de replantes, dado que es una especie más resistente a las bajas temperaturas que E. globulus. En cambio, Eucalyptus viminalis, a la misma edad, sólo logró cerca de $9 \mathrm{~m}^{3} /$ ha-año, lo que no es un resultado muy atractivo.

En general se puede indicar que el área de estudio se inserta en un mismo distrito climático con suelos de un mismo origen, con la única diferencia que pertenecen a dos series de suelo taxonómicamente distintas, pero de gran similitud. La falta de diferencias significativas en el crecimiento de Eucalyptus globulus entre las dos series, considerando que las plantaciones tuvieron técnicas de cultivo muy similares, indica que los suelos presentan características y propiedades dentro un rango que puede considerarse homogéneo desde el punto de vista de la fertilidad del suelo. Proba- 
BOSQUE 25(1): 95-101, 2004

Crecimiento de las plantaciones de Eucalyptus globulus sobre suelos rojo arcillosos...

\section{CUADRO 4}

Resultados para el caso especial de un rodal de Eucalyptus globulus y otras especies. Results for a particular case of a Eucalyptus globulus stand and other species.

\begin{tabular}{|c|l|c|c|c|c|c|}
\hline Rodal & Especie & $\begin{array}{c}\text { Superficie } \\
\text { (ha) }\end{array}$ & Edad & $\begin{array}{c}\text { Densidad } \\
(\mathrm{arb} . / \mathrm{ha})\end{array}$ & $\begin{array}{c}\text { Area Basal } \\
\left(\mathrm{m}^{2} / \mathrm{ha}\right)\end{array}$ & $\begin{array}{c}\text { IMA } \\
\left(\mathrm{m}^{3} / \mathrm{ha}\right)\end{array}$ \\
\hline 1 & E. globulus & 5,2 & 8 & 1.507 & 34,4 & 32,1 \\
2 & E. delegatensis & 4,5 & 8 & 1.528 & 29,1 & 18,1 \\
3 & E. viminalis & 12,0 & 8 & 1.096 & 15,1 & 8,9 \\
\hline
\end{tabular}

blemente sean más significativas las diferencias relacionadas con la posición topográfica, o el efecto del uso histórico de los suelos que las taxonómicas.

Muy decisivas para el resultado de las plantaciones fueron las técnicas de cultivo, que al optimizarlas dieron origen a un crecimiento considerablemente superior, de hasta tres veces el crecimiento en volumen. Este resultado es tan decisivo, que es necesario llevar a efecto una transferencia tecnológica entre los productores, para convencerlos de lo importante de este aspecto y obtener así volúmenes adecuados al momento de la cosecha. Sin embargo, deberá ser considerado también que la especie E. globulus debe ser establecida en sitios cuidadosamente seleccionados, evitando sitios en hondonadas y en exposiciones con mayor probabilidad de sufrir heladas en cantidad e intensidad.

Entre las otras especies medidas E. viminalis presenta un menor potencial de crecimiento que E. globulus; en cambio, E. delegatensis se muestra promisorio. E. nitens, especie que no fue medida en este trabajo, también es una buena alternativa, por su buen potencial de crecimiento: $20,5 \mathrm{~m}^{3} / \mathrm{ha}-$ año a los 4 años en la localidad de Tegualda (6).

Lo anterior permite proyectar a la especie Eucalyptus globulus en la zona de estudio, debiéndose tomar en consideración las recomendaciones aquí planteadas y, además, apoyar los programas de mejoramiento genético en sus esfuerzos por lograr razas de mayor resistencia al frío.

\section{CONCLUSIONES}

- El incremento medio anual de Eucalyptus globulus, medido en distintas plantaciones del área de estudio (en total 300 ha), alcanzó el nivel más bajo del crecimiento proyectado para la región (10-12 $\left.\mathrm{m}^{3} / \mathrm{ha}-\mathrm{año}\right)$, resultado que probablemente es producto, en primer lugar, de técnicas poco adecuadas en el establecimiento $\mathrm{y}$, en segundo lugar, originado por un factor climático: las temperaturas bajas en el período invernal.

- El potencial de Eucalyptus globulus, de 32,1 $\mathrm{m}^{3} /$ ha-año, medido en una plantación establecida con técnicas adecuadas y ubicada en un sitio favorable (5,2 ha), corresponde al crecimiento máximo proyectado para esta zona.

- Los resultados obtenidos con Eucalyptus globulus en sitios promedio indican que la especie está en una zona climática marginal desde el punto de vista de la temperatura invernal. Esto exige que la elección de sitios para establecerla en plantaciones debe ser cuidadosa, seleccionando aquellos que respondan mejor a sus requerimientos. En estos sitios es necesario, además, aplicar técnicas de establecimiento adecuadas al lugar, para lograr un resultado cercano al potencial, que es considerablemente superior al promedio actual.

- Otras especies de eucaliptos analizadas no son una alternativa real a Eucalyptus globulus en el área. E. viminalis presentó un rendimiento más bajo; E. delegatensis con crecimientos promisorios aún debe ser evaluada como especie comercial.

\section{BIBLIOGRAFIA}

(1) PRADO, J. A., S. BARROS. Eucalyptus. Principios de silvicultura y manejo. División Silvicultura, Instituto Forestal, CORFO, Santiago de Chile. 1989. 199 p. 
BOSQUE 25(1): 95-101, 2004 Crecimiento de las plantaciones de Eucalyptus globulus sobre suelos rojo arcillosos...

(2) INSTITUTO FORESTAL Especies forestales exóticas de interés económico para Chile. INFOR-CORFO, Santiago, Chile. 1986. 168 p.

(3) SCHLATTER, J. E., V. GERDING, H. HUBER. Sistema de ordenamiento de la Tierra. Herramienta para la planificación forestal, aplicado a la X Región. Serie Técnica, Fac. de Ciencias Forestales, Univ. Austral de Chile, Valdivia, Chile. 1995. 93 p.

(4) INSTITUTO DE INVESTIGACIONES AGRARIAS. Suelos volcánicos de Chile. Ed. Tosso, J., Santiago: INIA, Ministerio de Agricultura, 1985. 723 p.
(5) BAHAMONDEZ, C., M. FERRANDO, M. MARTIN, J. C. PINILLA. Determinación de funciones de volumen y razón de volumen para eucalipto. Proyecto Instituto Forestal/ CONICYT-FONDEF. Antecedentes biométricos y modelos de apoyo a la gestión y manejo racional del Eucaliptus. $1995.15 \mathrm{p}$

(6) APARICIO, J. L. Rendimiento y biomasa de Eucalyptus nitens con alternativas nutricionales para una silvicultura sustentable en un suelo rojo arcilloso. Tesis MSc., Fac. de Ciencias Forestales. Univ. Austral de Chile, Valdivia, Chile. 2001. 170 p. 